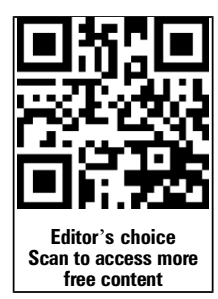

- Additional material is published online only. To view please visit the journal online (http://dx.doi.org/10.1136/ heartjnl-2015-307754).

${ }^{1}$ Department of Cardiology, Academic Medical Center Amsterdam, Amsterdam, The Netherlands ${ }^{2}$ Interuniversity Cardiology Institute of the Netherlands, The Netherlands

${ }^{3}$ Department of Cardiology, Leiden University Medical

Center, Leiden,

The Netherlands

${ }^{4}$ Department of Cardiology, Radboud University Medical Center Nijmegen, Nijmegen, The Netherlands

${ }^{5}$ Department of Cardiothoracic Surgery, Academic Medical Center Amsterdam,

Amsterdam, The Netherlands ${ }^{6}$ Department of Cardiothoracic Surgery, Leiden University Medical Center, Leiden, The Netherlands

\section{Correspondence to} Dr Berto J Bouma, Department of Cardiology, Academic Medical Center Amsterdam, Room B2-256, Meibergdreef 9, Amsterdam $1105 \mathrm{AZ}$, The Netherlands;

b.j.bouma@amc.uva.nl

Received 3 March 2015 Revised 14 June 2015 Accepted 18 June 2015 Published Online First 14 July 2015

\section{SLinked}

- http://dx.doi.org/10.1136/ heartjnl-2015-308294

\section{CrossMark}

$$
\begin{aligned}
& \text { To cite: Bokma JP, } \\
& \text { Winter MM, Oosterhof T, } \\
& \text { et al. Heart }
\end{aligned}
$$$$
\text { 2015:101:1717-1723. }
$$

\title{
Individualised prediction of pulmonary homograft durability in tetralogy of Fallot
}

\author{
Jouke P Bokma, ${ }_{1}^{1,2}$ Michiel M Winter, ${ }^{1}$ Thomas Oosterhof, ${ }^{1}$ Hubert W Vliegen, ${ }^{3}$ \\ Arie P van Dijk, ${ }^{4}$ Mark G Hazekamp, ${ }^{5,6}$ Dave R Koolbergen, ${ }^{5,6}$ Maarten Groenink, ${ }^{1}$ \\ Barbara JM Mulder, ${ }^{1,2}$ Berto J Bouma ${ }^{1,2}$
}

\begin{abstract}
Background In patients with repaired tetralogy of Fallot (rTOF), multiple reoperations or percutaneous interventions after pulmonary valve replacement (PVR) may be necessary due to limited homograft durability. However, data to guide individualised prediction of homograft durability remain scarce. The aim of this study was to provide risk models for RV to pulmonary artery homograft durability.
\end{abstract}

Methods This retrospective multicentre study included consecutive patients with rTOF who had undergone PVR at an age of $>12$ years. Homograft dysfunction was defined as at least moderate pulmonary regurgitation (PR) or pulmonary stenosis (PS) (pressure gradient $\geq 36 \mathrm{~mm} \mathrm{Hg}$ ) as assessed by echocardiography.

Reintervention was defined as percutaneous intervention or redo-PVR.

Results A total of 153 patients with rTOF were

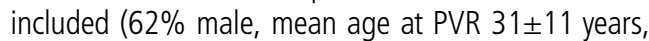
pulmonary homograft 96\%, follow-up 9.6 years (IQR $5.9,13.3)$ ). Average freedom from homograft dysfunction and reintervention after 10 years was $74 \%$ and $89 \%$, respectively. In multivariable Cox proportional hazards analysis, postoperative PS $\geq 20 \mathrm{~mm} \mathrm{Hg}$ (HR 6.52, 95\% Cl 3.09 to 13.7), postoperative PR $\geq$ grade 1 (HR 3.13, $95 \% \mathrm{Cl} 1.45$ to 6.74$)$ and age at PVR $<18$ years (HR 3.52, 95\% Cl 1.64 to 7.53) were independently predictive for homograft dysfunction. In patients without any risk factor, 10-year freedom from homograft dysfunction and reintervention was excellent (91\% and $96 \%$, respectively) in contrast to patients with $\geq 2$ risk factors ( $25 \%$ and $73 \%$, respectively).

Conclusions Individualised prediction of homograft durability in patients with rTOF can be guided by early postoperative echocardiography. In adult patients without early postoperative PS or PR, homograft dysfunction and reintervention are unlikely to occur within 10 years, and follow-up may be less stringent.

\section{INTRODUCTION}

Tetralogy of Fallot (TOF) is the most common form of cyanotic congenital heart disease, and occurs in 4-5 of 10000 live births. ${ }^{1}$ Advances in the early management have led to a survival of over $95 \%$ into adulthood. ${ }^{2}$ However, longstanding RV overload due to pulmonary regurgitation (PR) after cardiac surgery may lead to RV dysfunction and subsequently heart failure, sustained ventricular tachycardia and sudden cardiac death. ${ }^{3} 4$ Consequently, morbidity and mortality rise substantially from the third decade of life in patients with repaired TOF (rTOF). ${ }^{5}$ Pulmonary valve replacement (PVR) can be performed by implantation of several types of RV outflow tract conduits, usually bioprosthetic valves or homografts. ${ }^{67}$ Early beneficial effects after PVR include a reduction in RV volumes, a decrease of PR and an improvement in New York Heart Association functional class. ${ }^{689}$ However, a major concern after PVR is the need for future reoperations or reinterventions due to bioprosthetic conduit or homograft dysfunction. $^{6} \quad 7 \quad 9{ }^{10}$ Average RV outflow tract conduit lifespan has been estimated about 10-15 years. ${ }^{9} 11 \quad 12$ While most studies reported time to redo-PVR or reintervention, the occurrence of homograft dysfunction may be a more sensitive precursor of lifespan. Consequently, previous studies failed to identify sufficient risk factors to allow individualised estimation of homograft durability as the number of patients with reintervention was insufficient. $^{6} 713$ Furthermore, inclusion of young children has severely impacted average homograft lifespan in several studies as their conduits or homografts were likely to deteriorate more rapidly. ${ }^{6} 14$ Recently, percutaneous pulmonary valve implantation has emerged as an alternative for surgical PVR with excellent short-term and medium-term results. ${ }^{15}$ However, percutaneous implantation is currently only feasible in patients with rTOF and having appropriate RV outflow tract anatomy, ${ }^{15}$ leaving surgical PVR the method of choice for many patients. Better prediction of surgical RV outflow tract homograft dysfunction and reintervention in patients with rTOF can help to determine adequate outpatient follow-up intervals, therewith limiting healthcare burden and costs. The objective of our study was to determine long-term homograft durability after PVR, as well as factors prognostic for early homograft dysfunction and reintervention.

\section{METHODS}

In this retrospective, multicentre study, 153 out of a total of 157 consecutive patients with rTOF who had undergone PVR between 1986 and 2013 were included. The excluded patients underwent PVR with a Contegra valved conduit instead of a homograft. Three large tertiary centres (Academic Medical Center Amsterdam, Leiden University Medical Center and Radboud University Medical Center Nijmegen) participated in the study. All patients were at least 12 years old at the time of PVR. In patients who had undergone subsequent 
PVRs, only the first PVR was included for analysis. Medical records were reviewed for all patients. Follow-up was completed until the date of last medical contact or death. Data collected included patient characteristics, operations and concomitant procedures. In addition, preoperative echocardiographic, cardiovascular magnetic resonance (CMR) and ECG examinations were collected. Homograft function after PVR was analysed by serial postoperative echocardiographic examinations. This retrospective study was conducted in accordance with all human research regulatory guidelines, and the need to obtain informed consent was waived by the institutional ethics committee.

\section{Pulmonary valve replacement}

Current international guidelines recommend PVR in symptomatic patients with rTOF and severe PR or pulmonary stenosis (PS). ${ }^{12}{ }^{16}$ In addition, PVR should be considered in the presence of progressive RV enlargement, RV dysfunction, tricuspid regurgitation (TR) or (supra-)ventricular arrhythmias. ${ }^{12} 16$ These guidelines formed the basis for local heart team policy, although exact interpretation of these and previous guidelines may have differed in detail. All homografts were allocated by Bio Implant Services, Leiden, the Netherlands, from where the data on homograft diameter were obtained. All patients were operated on with normothermic or moderately hypothermic cardiopulmonary bypass. Aortic cross-clamping was dependent on the surgeon's preference. When no transannular patch was used in the past, the pulmonary artery was opened longitudinally, and if necessary, this incision was extended across the pulmonary annulus into the RV outflow tract. Homografts were inserted in the orthotopic pulmonary position with one proximal and one distal end-to-end running suture. If a transannular patch was used in the primary correction, the patch material was resected as much as possible, after which, the RV outflow tract was reefed as far as needed to match the homograft size. Concomitant surgical procedures were performed as clinically indicated.

\section{Echocardiography}

Echocardiographic examination was performed on local available echocardiography systems. Data on the last echocardiographic assessment before PVR were acquired. In addition, data on serial echocardiographic assessments after PVR were acquired. The first postoperative echocardiogram obtained was the last before hospital discharge or alternatively the first during outpatient follow-up to exclude variation in RV outflow tract gradients due to differences in cardiac output perioperatively. Peak systolic pressure gradient across the RV outflow tract homograft was estimated by continuous wave Doppler echocardiography, using the modified Bernoulli equation. Pressure gradients of $20-35 \mathrm{~mm} \mathrm{Hg}$ and $\geq 36 \mathrm{~mm} \mathrm{Hg}$ were considered as mild and moderate PS, respectively. ${ }^{12} 17$ Colour flow mapping of the RV outflow tract and branch pulmonary arteries was used to grade the PR into four categories: 0 , absent/trivial; 1 , mild; 2 , moderate; 3 , severe. ${ }^{12} 18$ In addition, echocardiographic derived grade $(0-3)$ of TR was determined by vena contracta width, and estimation of RV systolic pressures by TR jet gradient using the modified Bernoulli equation. ${ }^{18}$ Pulmonary artery pressures were estimated by subtracting maximum gradient across the pulmonary valve from RV systolic pressure (estimated right atrial pressure plus TR jet gradient).

\section{Cardiovascular magnetic resonance}

CMR imaging was performed on locally available magnetic resonance systems. CMR studies were performed with the use of local imaging protocols as described previously. ${ }^{8}$ The last preoperative CMR was analysed at local centres. Scout images were obtained in three orthogonal planes, and standard two-chamber and four-chamber views were acquired. From these views, a stack of 12 to 14 slices was acquired in the short-axis orientation (steady state free precession) to calculate both RV and LV volumes from endocardial contours with the use of Mass software (Medis, Leiden, the Netherlands). All volumes were indexed for body surface area. The EF was calculated as the end-diastolic volume minus the end-systolic volume (which equals the stroke volume) divided by the end-diastolic volume and expressed as percentage. Velocity mapping was performed with the use of velocity-encoded phase contrast sequence. PR fraction was calculated as PR flow divided by systolic forward flow and expressed as a percentage.

\section{Definitions of outcome}

The outcome measures were RV outflow tract homograft dysfunction and reintervention after PVR in patients with rTOF. Homograft dysfunction was defined as: at least moderate PS (peak systolic pressure gradient $\geq 36 \mathrm{~mm} \mathrm{Hg}$ ) or PR (grade 2 or higher) on echocardiography. ${ }^{12} 1718$ Reintervention was defined as balloon dilation, redo-PVR or percutaneous Melody implantation.

\section{Statistical analysis}

Data are described as number with frequency, median with IQR and mean with SD. Univariate Cox proportional hazard analysis was used to identify predictors for homograft dysfunction after PVR. Continuous variables predictive for homograft dysfunction were dichotomised on relevant cut-off values by plotting receiver operating characteristic curves. Multivariable analysis was performed, including all variables with a $\mathrm{p}$ value below 0.05 in univariate analysis. Multivariable analysis was performed with a forward conditional algorithm. The assumption of proportional hazards was, first, checked by log-minus-log plots for each predictive variable and, second, by adding a timedependent covariate in the Cox proportional hazards model. A risk model was constructed using all independently predictive variables for homograft dysfunction. Kaplan-Meier curves were constructed to determine actual freedom from homograft dysfunction for each risk category in the risk model. Yearly risk was estimated by dividing the total number of events by the total patient years at risk for each risk category. The C-statistic of the multivariate model reflects the area under the curve for binary censored survival data. Changes in haemodynamic parameters early after PVR were analysed with paired Student's t test for normal distributed variables and with the Wilcoxon signed-rank test for non-normal distributed variables. Analyses were performed with SPSS V.20.0 (IBM, Armonk, New York, USA) and R statistics V.2.13.1 (R Foundation for Statistical Computing, Vienna, Austria). A p value $<0.05$ was considered statistically significant.

\section{RESULTS}

\section{Patient population}

Our population consisted of 153 patients with rTOF (62\% male). Median follow-up after PVR was 9.6 years (IQR 5.9, 13.3). Patient and surgical characteristics are listed in table 1. Early postoperative echocardiography was available in 140 $(89 \%)$ patients, whereas serial late postoperative echocardiographic follow-up examinations were acquired in all patients. Data on preoperative CMR were available in $105(67 \%)$ patients; in the remaining patients, CMR examinations were not 
Table 1 Patient and surgical characteristics

\begin{tabular}{|c|c|}
\hline Number of patients & 153 \\
\hline Follow-up after PVR (years) & $9.6(5.9,13.3)$ \\
\hline Male gender & $95(62 \%)$ \\
\hline Age at PVR (years) & $30.8 \pm 11$ \\
\hline \multicolumn{2}{|l|}{ Initial correction } \\
\hline Myectomy/valvulotomy & $17(11 \%)$ \\
\hline RV patch & $21(14 \%)$ \\
\hline Transannular patch & $106(69 \%)$ \\
\hline Other & $9(6 \%)$ \\
\hline Age at initial correction (years) & $4.8(2.4,7.5)$ \\
\hline Previous shunt procedure & $73(48 \%)$ \\
\hline Waterston & $31(42 \%)$ \\
\hline Potts & $6(8 \%)$ \\
\hline Blalock-Taussig & $32(44 \%)$ \\
\hline Other & $4(5 \%)$ \\
\hline Age at shunt procedure (years) & $1.5(0.7,2.9)$ \\
\hline Down syndrome & $4(3 \%)$ \\
\hline $22 q 11$ deletion & $5(3 \%)$ \\
\hline Other cause of mental retardation & $8(5 \%)$ \\
\hline \multicolumn{2}{|l|}{ History prior to PVR } \\
\hline Ventricular tachycardia & $15(10 \%)$ \\
\hline Supraventricular tachycardia & $21(14 \%)$ \\
\hline Heart failure & $6(4 \%)$ \\
\hline QRS duration before PVR (ms) & $152 \pm 27$ \\
\hline NYHA class $\geq$ II before PVR & $67 / 119(56 \%)$ \\
\hline \multicolumn{2}{|l|}{ Indication for PVR } \\
\hline Pulmonary regurgitation & $130(85 \%)$ \\
\hline Other & $23(15 \%)$ \\
\hline \multicolumn{2}{|l|}{ Surgical data } \\
\hline \multicolumn{2}{|l|}{ Type of RV outflow tract homograft } \\
\hline Pulmonary homograft & $147(96 \%)$ \\
\hline Aortic homograft & $6(4 \%)$ \\
\hline Previous PVR & $2(1 \%)$ \\
\hline \multicolumn{2}{|l|}{ Concomitant procedures } \\
\hline Tricuspid valve plasty or ring & $29(19 \%)$ \\
\hline RV aneurysm/patch resection & $52(34 \%)$ \\
\hline Pulmonary artery angioplasty & $34(22 \%)$ \\
\hline Ventricular septal defect closure & $9(6 \%)$ \\
\hline Diameter homograft (mm) & $25.2 \pm 2.0$ \\
\hline
\end{tabular}

performed due to contraindications for CMR or because CMR imaging was not yet available. During follow-up, three patients developed homograft endocarditis; all of these patients also had homograft dysfunction. Five patients died during follow-up after PVR. Three patients died after progressive heart failure, and two died suddenly. One patient had graft dysfunction at the time of death.

\section{Early haemodynamic changes after PVR}

Echocardiographic and CMR parameters before and early after PVR are listed in table 2. Briefly, PVR led to a marked reduction of both echocardiographic PR grade and CMR-derived PR fraction. CMR-derived RV volumes decreased. In addition, small non-significant increases in RV EF $(p=0.07)$ and LV EF $(p=0.15)$ occurred after PVR. The maximum early postoperative homograft gradient was $19 \mathrm{~mm} \mathrm{Hg}$ (SD 12), and in $88 \%$ of patients, postoperative PR was not more than trivial.

\section{Homograft dysfunction}

During long-term follow-up after PVR, homograft dysfunction occurred in 39 patients (PS in 29 patients, PR in 10 patients). Freedom from homograft dysfunction was $82 \%$ after 5 years, $74 \%$ after 10 years and 68\% after 15 years (figure 1). In univariate analysis (table 3 ), several preoperative and early postoperative parameters were predictive for homograft dysfunction. Multivariable analysis revealed that postoperative PS $\geq 20 \mathrm{~mm} \mathrm{Hg}$ (HR 6.52, 95\% CI 3.09 to $13.7, \mathrm{p}<0.001$ ), postoperative $\mathrm{PR} \geq$ grade 1 (HR 3.13, 95\% CI 1.45 to 6.74 , $\mathrm{p}=0.004$ ) and age at PVR $<18$ years (HR 3.52, 95\% CI 1.64 to $7.53, \mathrm{p}=0.001)$ remained independently predictive for homograft dysfunction (table 3). Era of PVR was predictive for homograft dysfunction in univariate, but not in multivariable, analysis $(p=0.13)$. A risk model was constructed containing all three independent predictors for homograft dysfunction. In patients without any risk factor (low risk), freedom from homograft dysfunction was $91 \%$ after 10 years (figure 2). On the contrary, in patients with at least two out of three risk factors (high risk), freedom from homograft dysfunction was only $25 \%$ after 10 years. The yearly risk for homograft dysfunction was $0.7 \%$ in low-risk patients as compared with $5.8 \%$ in intermediate-risk and $17 \%$ in high-risk patients. The C-statistic of the final multivariable risk model was 0.85 (95\% CI 0.77 to $0.92, \mathrm{p}<0.001)$. Homograft diameter was similar in patients who had undergone PVR at an age $<18$ years $(24.6 \pm 2.2 \mathrm{~mm}))$ and those $>18$ years at PVR $(25.2 \pm 2.0 \mathrm{~mm}))(\mathrm{p}=0.31)$. Patients operated after 2005 $(n=58)$ had similar postoperative PS $(p=0.31)$, but lower PR grade $(\mathrm{p}=0.03)$.

In addition, we repeated our analysis in the subgroup of patients $(n=147)$ in whom a pulmonary homograft was used for PVR to determine whether homograft type influenced the risk factors for dysfunction in the first analysis. In multivariable analysis, postoperative PS $\geq 20 \mathrm{~mm} \mathrm{Hg}$ (HR 5.56, 95\% CI 2.55 to 12.1, $\mathrm{p}<0.001$ ), postoperative $\mathrm{PR} \geq$ grade 1 (HR 3.42, 95\% CI 1.56 to $7.50, \mathrm{p}=0.002$ ) and age at $\mathrm{PVR}<18$ years (HR 2.86, $95 \%$ CI 1.16 to $7.06, \mathrm{p}=0.02$ ) all remained independently predictive for pulmonary homograft dysfunction.

\section{Homograft reintervention}

Reintervention was performed in 16 patients (redo-PVR in 13 patients, Melody valve implantation in one patient and balloon dilation of homograft in two patients). Both patients with a balloon dilation of the homograft eventually also underwent redo-PVR due to secondary PR. In all 16 patients, homograft dysfunction occurred prior to reintervention, which was performed at a median of 0.4 years after the onset of homograft dysfunction (IQR 0.01-2.0) and within 6 months after onset of homograft dysfunction in eight patients. The remaining 23 patients with homograft dysfunction have been managed conservatively for a median of 8.4 years (IQR 2.6-9.7). All patients who were managed conservatively for homograft dysfunction had PS $<64 \mathrm{~mm} \mathrm{Hg}$ at the time of homograft dysfunction, and none had severe PR (see online supplementary e-table 1). In addition, conservatively managed patients had better preserved RV end-diastolic volume and RV EF on CMR, if available, compared with those who underwent reintervention (see online supplementary e-table 1 ).

In the entire study population, freedom from homograft reintervention was $94 \%$ after 5 years, $89 \%$ after 10 years and $89 \%$ after 15 years (figure 1). Our proposed risk model was applied to determine freedom from homograft reintervention for each risk category. Freedom from homograft reintervention after 
Table 2 Imaging parameters

\begin{tabular}{|c|c|c|c|c|}
\hline Echocardiography & $\begin{array}{l}\text { Before PVR } \\
n=135\end{array}$ & $\begin{array}{l}\text { Early after PVR } \\
n=140\end{array}$ & $\begin{array}{l}\Delta \text { PVR } \\
n=125\end{array}$ & $\begin{array}{l}\mathrm{p} \text { Value } \Delta \\
\mathrm{n}=125\end{array}$ \\
\hline Time echo from PVR (years) & $-0.37(-0.14,-0.71)$ & $0.02(0.01,0.30)$ & & \\
\hline PR grade $(0-3)$ & & & $-2(-2$ to -3$)$ & $<0.001$ \\
\hline None/trivial (grade 0) & $3(2 \%)$ & $118(88 \%)$ & & \\
\hline Mild (grade 1) & $16(12 \%)$ & $14(10 \%)$ & & \\
\hline Moderate (grade 2) & $44(33 \%)$ & $1(1 \%)$ & & \\
\hline Severe (grade 3) & $70(53 \%)$ & $1(1 \%)$ & & \\
\hline PS-max gradient $(\mathrm{mm} \mathrm{Hg})$ & $24 \pm 21$ & $19 \pm 12$ & $-4.7 \pm 22$ & 0.01 \\
\hline TR grade $(0-3)$ & & & $0(-1$ to 0$)$ & $<0.001$ \\
\hline None/trivial (grade 0) & $2(2 \%)$ & $23(18 \%)$ & & \\
\hline Mild (grade 1) & $85(69 \%)$ & $96(71 \%)$ & & \\
\hline Moderate (grade 2) & $26(21 \%)$ & $15(11 \%)$ & & \\
\hline Severe (grade 3) & $10(8 \%)$ & 0 & & \\
\hline Estimated PAP & $20 \pm 15$ & $19 \pm 13$ & $-1 \pm 16$ & 0.64 \\
\hline CMR & $n=101$ & $n=81$ & $n=67$ & $n=67$ \\
\hline Time CMR from PVR (years) & $-0.46(-0.29,-0.96)$ & $0.88(0.51,1.60)$ & & \\
\hline RV EDVI $\left(\mathrm{mL} / \mathrm{m}^{2}\right)$ & $171 \pm 40$ & $116 \pm 31$ & $-55 \pm 31$ & $<0.001$ \\
\hline RV ESVI $\left(\mathrm{mL} / \mathrm{m}^{2}\right)$ & $99 \pm 31$ & $66 \pm 29$ & $-33 \pm 24$ & $<0.001$ \\
\hline LV EDVI $\left(\mathrm{mL} / \mathrm{m}^{2}\right)$ & $89 \pm 21$ & $97 \pm 21$ & $8 \pm 18$ & $<0.001$ \\
\hline LV ESVI $\left(\mathrm{mL} / \mathrm{m}^{2}\right)$ & $43 \pm 14$ & $46 \pm 16$ & $3.0 \pm 12$ & 0.04 \\
\hline RV EF (\%) & $43 \pm 9$ & $45 \pm 10$ & $2.0 \pm 9.0$ & 0.07 \\
\hline LV EF (\%) & $52 \pm 11$ & $53 \pm 11$ & $1.4 \pm 7.5$ & 0.15 \\
\hline PR fraction (\%) & $46 \pm 11$ & $0(0,4)$ & $-42 \pm 12$ & $<0.001$ \\
\hline
\end{tabular}

10 years was $96 \%$ in low-risk patients, $83 \%$ in intermediate-risk patients and $75 \%$ in high-risk patients $(\mathrm{p}=0.004)$ (figure 3$)$.

\section{DISCUSSION}

We have demonstrated that pulmonary homograft durability can be accurately predicted after postoperative echocardiography in patients with rTOF. In our contemporary cohort, homograft

\section{Homograft dysfunction and reintervention after PVR}

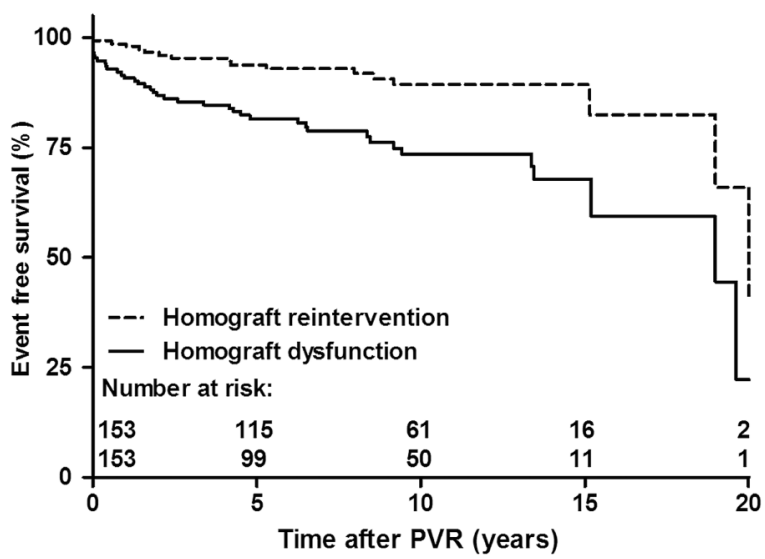

Figure 1 Homograft dysfunction and reintervention after PVR. Kaplan-Meier curves displaying freedom from homograft dysfunction (pulmonary stenosis $\geq 36 \mathrm{~mm} \mathrm{Hg}$ or moderate pulmonary regurgitation) and freedom from homograft reintervention (redo-PVR, Melody implantation or balloon dilation of homograft) after PVR. PVR, pulmonary valve replacement. dysfunction and reintervention after PVR occurred less rapidly than previously reported, ${ }^{6} 71013141920$ and was unlikely to occur within 10 years in adult patients without early postoperative PS or PR.

\section{Homograft dysfunction and reintervention}

Several factors may explain our low rates of homograft dysfunction and reintervention when compared with previous reports. First, we only included patients older than 12 years at PVR as adult-sized homograft implantation is usually feasible in these patients. In younger children, a progressive mismatch between annulus size and homograft diameter ${ }^{71-23}$ has been reported to contribute to early homograft reinterventions. In our cohort, children between 12 and 18 years old were also at risk for homograft dysfunction despite equally sized homografts when compared with adults. We hypothesise that a more active immune system contributes to more rapid homograft degeneration in these adolescents. ${ }^{24}$ Second, we observed lower incidence of homograft dysfunction in more recently operated patients. This may be due to better selection of patients, better preservation techniques and the increasing experience of surgeons in performing PVR. ${ }^{25}$ Indeed, we found less postoperative PR in patients operated after 2005. Furthermore, the use of intraoperative echocardiography may assist in positioning of the RV outflow tract homograft, leading to less postoperative PS and PR and increasing durability. Finally, homografts were used as an RV outflow tract conduit in all patients. The use of pulmonary and aortic homografts is more common in Europe while in most adults in North America, xenografts are used. Direct comparison between pulmonary and aortic homograft durability was not possible due to the low number of aortic homografts used 
Table 3 Cox proportional hazards regression for homograft dysfunction

\begin{tabular}{|c|c|c|c|c|c|c|}
\hline & \multicolumn{3}{|c|}{ Univariate } & \multicolumn{3}{|c|}{ Multivariable } \\
\hline & HR & $95 \% \mathrm{Cl}$ & p Value & HR & $95 \% \mathrm{Cl}$ & p Value \\
\hline \multicolumn{7}{|l|}{ Patient and surgical characteristics } \\
\hline Age at PVR (per year) & 0.96 & 0.93 to 0.99 & 0.03 & & & \\
\hline Age at PVR $<18$ years & 2.53 & 1.27 to 5.03 & 0.008 & 3.52 & 1.64 to 7.53 & 0.001 \\
\hline Era PVR (per year) & 0.93 & 0.87 to 0.99 & 0.02 & 0.94 & 0.87 to 1.02 & 0.13 \\
\hline Aortic homograft (vs pulmonary) & 2.52 & 0.82 to 7.78 & 0.11 & & & \\
\hline Diameter homograft (per mm) & 0.91 & 0.76 to 1.08 & 0.28 & & & \\
\hline \multicolumn{7}{|l|}{ Preoperative echocardiography } \\
\hline PS max gradient (per mm $\mathrm{Hg}$ ) & 1.01 & 0.99 to 1.02 & 0.35 & & & \\
\hline PR grade (per grade) & 1.11 & 0.69 to 1.77 & 0.67 & & & \\
\hline TR grade (per grade) & 1.37 & 0.77 to 2.43 & 0.29 & & & \\
\hline Estimated PAP (per mm Hg) & 1.01 & 0.98 to 1.04 & 0.51 & & & \\
\hline \multicolumn{7}{|l|}{ Postoperative echocardiography } \\
\hline PS max gradient (per mm Hg) & 1.04 & 1.03 to 1.05 & $<0.001$ & & & \\
\hline PS max gradient $\geq 20 \mathrm{~mm} \mathrm{Hg}$ & 5.43 & 2.64 to 11.1 & $<0.001$ & 6.52 & 3.09 to 13.7 & $<0.001$ \\
\hline PR grade (per grade) & 2.89 & 1.77 to 4.74 & $<0.001$ & & & \\
\hline$P R$ grade $\geq 1$ & 3.40 & 1.58 to 7.31 & 0.002 & 3.13 & 1.45 to 6.74 & 0.004 \\
\hline TR grade (per grade) & 1.44 & 0.72 to 2.88 & 0.31 & & & \\
\hline Estimated PAP (per mm Hg) & 1.00 & 0.94 to 1.04 & 0.96 & & & \\
\hline \multicolumn{7}{|l|}{ Preoperative CMR } \\
\hline RV EF (per \%) & 1.03 & 0.97 to 1.09 & 0.35 & & & \\
\hline LV EF (per \%) & 1.02 & 0.97 to 1.06 & 0.50 & & & \\
\hline RV EDVI (per $\mathrm{mL} / \mathrm{m}^{2}$ ) & 1.00 & 0.98 to 1.01 & 0.40 & & & \\
\hline LV EDVI (per mL/m²) & 1.01 & 0.99 to 1.03 & 0.63 & & & \\
\hline PR fraction (per \%) & 1.00 & 0.97 to 1.04 & 0.88 & & & \\
\hline
\end{tabular}

in our population. Earlier studies have reported better performance when homografts were implanted in their anatomical position. $^{21} 26$ However, homograft type did not significantly

Risk model for homograft dysfunction after PVR

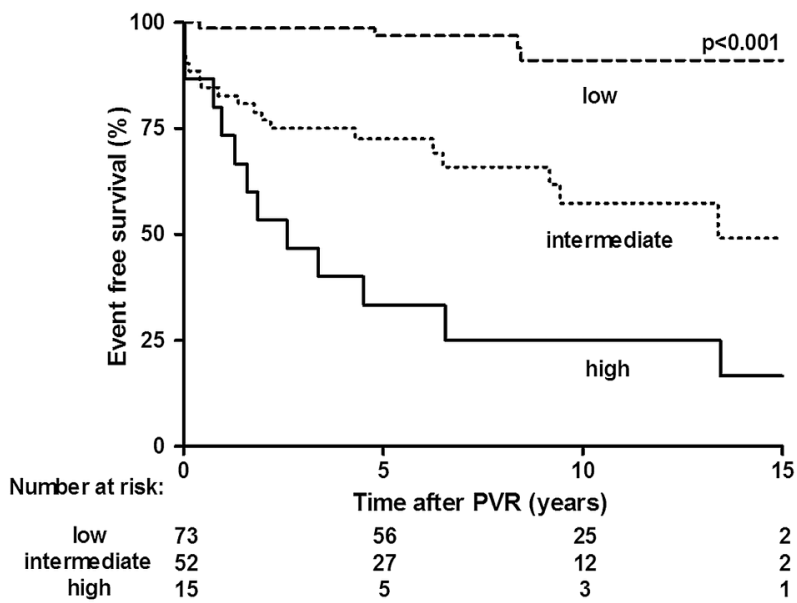

Figure 2 Risk model for homograft dysfunction after PVR. Freedom from homograft dysfunction in patients $(n=140)$ in whom early postoperative echocardiography was available. Three risk factors are incorporated in the risk model: (1) postoperative PS $\geq 20 \mathrm{~mm} \mathrm{Hg}$, (2) postoperative $P R$ grade $\geq 1$ and (3) age at PVR $<18$ years. Low-risk patients had no risk factors, intermediate had one risk factor and high had $\geq 2$ risk factors. PR, pulmonary regurgitation; PS, pulmonary stenosis; PVR, pulmonary valve replacement. influence the impact of the independent risk factors on homograft dysfunction as results from a second multivariable analysis, excluding patients with an aortic homograft, were comparable.

Risk model for homograft reintervention after PVR

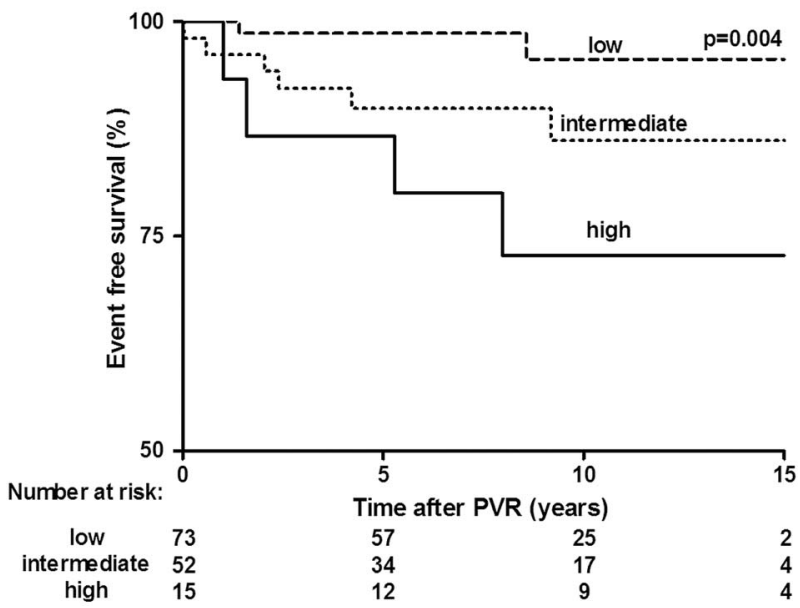

Figure 3 Risk model for homograft reintervention after PVR. Freedom from homograft dysfunction in patients $(n=140)$ in whom early postoperative echocardiography was available. Three risk factors are incorporated in the risk model: (1) postoperative PS $\geq 20 \mathrm{~mm} \mathrm{Hg}$, (2) postoperative $P R$ grade $\geq 1$ and (3) age at PVR $<18$ years. Low-risk patients had no risk factors, intermediate had one risk factor and high had $\geq 2$ risk factors. PR, pulmonary regurgitation; PS, pulmonary stenosis; PVR, pulmonary valve replacement. 


\section{Management of homograft dysfunction}

Most assuring in our study was the excellent homograft durability (91\% freedom from homograft dysfunction after 10 years) in patients without any risk factors. As several patients were managed conservatively for a dysfunctional homograft, the number of patients with homograft reintervention was lower. Previous studies reported variable rates of homograft or biopros-

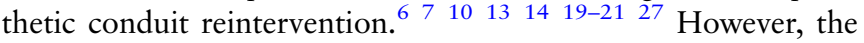
timing of reintervention can vary between institutions, which obscures comparison of the rate of conduit or homograft reintervention between different studies. Current guidelines advise reintervention in symptomatic patients with significant PS or PR after PVR. ${ }^{12} 16$ In asymptomatic patients, reintervention may be considered in patients with arrhythmias or deterioration of exercise capacity or haemodynamic parameters. ${ }^{12}$ In our cohort, reintervention was usually performed after PR or PS progressed to severe, and no patients were managed conservatively for severe PR or PS. However, patients who underwent reintervention also had worse haemodynamic parameters on CMR compared with those who were managed conservatively for a dysfunctional homograft.

We suspect eventually most patients with homograft dysfunction will require a reintervention due to ongoing homograft calcification and deterioration. Indeed, our risk model, which incorporated variables predictive for homograft dysfunction, was also prognostic for homograft reintervention.

\section{Risk stratification}

Stringent follow-up of cardiac and homograft function by CMR and echocardiography is warranted in patients with rTOF at risk for homograft dysfunction to guide timing of reinterventions. However, stringent follow-up may lead to an unnecessary rise in healthcare costs when applied to all patients. ${ }^{28}$ Individualised prediction of pulmonary homograft durability can help determine adequate follow-up intervals. With the implementation of our proposed risk models, follow-up in patients without risk factors can be less stringent. However, patients and physicians still need to be aware of other complications, which may occur in patients with rTOF, such as endocarditis or arrhythmias. Finally, percutaneous Melody valve implantation in a dysfunctional homograft has emerged as an alternative for redo-PVR, and excellent short-term and medium-term outcomes have been published. ${ }^{15}$ The use of percutaneous implanted valve has the potential to reduce the number of reoperations for patients with rTOF, and we eagerly await long-term results.

\section{Limitations}

The limitations of this study are mainly due to its retrospective design. First, in our cohort, patients were operated on in a long period, in which, techniques and timing of PVR have evolved. Second, preoperative echocardiography and CMR imaging data were missing in some patients, and were performed according to local protocols. Finally, we did not investigate the influence of donor characteristics or immunological variables on the occurrence of homograft durability. However, in previous studies, these factors were not predictive for homograft reintervention. 713

\section{CONCLUSION}

In the current era, pulmonary homograft durability after PVR in rTOF has improved dramatically when compared with earlier reports. We demonstrated that younger age, early postoperative PS and PR were predictive for early homograft dysfunction and reintervention. Our findings guide risk stratification for homograft durability, and can improve determination of outpatient follow-up intervals.

\section{Key messages}

What is already known on this subject?

Patients with Tetralogy of Fallot may require pulmonary valve replacement to correct residual pulmonary regurgitation after corrective surgery in childhood. However, these patients may require multiple reoperations due to limited $\mathrm{RV}$ outflow tract homograft durability.

\section{What might this study add?}

Individualised prediction of homograft durability can be guided by early postoperative echocardiography. Mild pulmonary stenosis and regurgitation and younger age are predictive for early homograft dysfunction in patients with tetralogy of Fallot.

\section{How might this impact on clinical practice?}

Physicians should use early postoperative echocardiography to predict expected homograft durability and determine adequate outpatient follow-up intervals to assess haemodynamic parameters.

Contributors All authors contributed in the conception, design, critical revision and final approval of this manuscript. JPB analysed and interpreted the data and drafted the manuscript under the supervision of senior authors, BJMM and BJB.

Funding This work was supported by the Parelsnoer Institute and NutsOhra foundation.

Competing interests None declared.

Ethics approval Institutional Ethics Committee of the Academic Medical Center.

Provenance and peer review Not commissioned; externally peer reviewed.

\section{REFERENCES}

1 Van der Bom T, Bouma BJ, Meijboom FJ, et al. The prevalence of adult congenital heart disease, results from a systematic review and evidence based calculation. Am Heart J 2012;164:568-75.

2 D'Udekem Y, Galati JC, Rolley GJ, et al. Low risk of pulmonary valve implantation after a policy of transatrial repair of tetralogy of Fallot delayed beyond the neonatal period: the Melbourne experience over 25 years. J Am Coll Cardiol 2014;63:563-8.

3 Valente AM, Gauvreau K, Assenza GE, et al. Contemporary predictors of death and sustained ventricular tachycardia in patients with repaired tetralogy of Fallot enrolled in the INDICATOR cohort. Heart 2014;100:247-53.

4 Knauth AL, Gauvreau K, Powell AJ, et al. Ventricular size and function assessed by cardiac MRI predict major adverse clinical outcomes late after tetralogy of Fallot repair. Heart 2008;94:211-16.

5 Nollert G, Fischlein T, Bouterwek $\mathrm{S}$, et al. Long-term survival in patients with repair of tetralogy of Fallot: 36-year follow-up of 490 survivors of the first year after surgical repair. J Am Coll Cardiol 1997;30:1374-83.

6 Lee $\mathrm{C}$, Kim YM, Lee $\mathrm{C}-\mathrm{H}$, et al. Outcomes of pulmonary valve replacement in 170 patients with chronic pulmonary regurgitation after relief of right ventricular outflow tract obstruction: implications for optimal timing of pulmonary valve replacement. J Am Coll Cardiol 2012;60:1005-14.

7 Oosterhof T, Meijboom FJ, Vliegen HW, et al. Long-term follow-up of homograft function after pulmonary valve replacement in patients with tetralogy of Fallot. Eur Heart J 2006;27:1478-84.

8 Oosterhof T, van Straten A, Vliegen HW, et al. Preoperative thresholds for pulmonary valve replacement in patients with corrected tetralogy of Fallot using cardiovascular magnetic resonance. Circulation 2007;116:545-51.

9 Ferraz Cavalcanti PE, Sá MPBO, Santos CA, et al. Pulmonary valve replacement after operative repair of tetralogy of Fallot: meta-analysis and meta-regression of 3,118 patients from 48 studies. J Am Coll Cardiol 2013;62:2227-43. 
10 Van de Woestijne PC, Mokhles MM, de Jong PL, et al. Right ventricular outflow tract reconstruction with an allograft conduit in patients after tetralogy of Fallot correction: long-term follow-up. Ann Thorac Surg 2011;92:161-6.

11 Geva T. Indications for pulmonary valve replacement in repaired tetralogy of Fallot the quest continues. Circulation 2013;128:1855-7.

12 Baumgartner $\mathrm{H}$, Bonhoeffer $\mathrm{P}$, Groot NMSD, et al. ESC guidelines for the management of grown-up congenital heart disease (new version 2010). Eur Heart $J$ 2010;31:2915-57.

13 Troost E, Meyns B, Daenen W, et al. Homograft survival after tetralogy of Fallot repair: determinants of accelerated homograft degeneration. Eur Heart $J$ 2007;28:2503-9.

14 Brown JW, Ruzmetov M, Rodefeld MD, et al. Right ventricular outflow tract reconstruction with an allograft conduit in non-ross patients: risk factors for allograft dysfunction and failure. Ann Thorac Surg 2005;80:655-64.

15 McElhinney DB, Hellenbrand WE, Zahn EM, et al. Short- and medium-term outcomes after transcatheter pulmonary valve placement in the expanded multicenter US melody valve trial. Circulation 2010;122:507-16.

16 Warnes CA, Williams RG, Bashore TM, et al. ACC/AHA 2008 guidelines for the management of adults with congenital heart disease a report of the American College of Cardiology/American Heart Association Task Force on Practice Guidelines (Writing Committee to Develop Guidelines on the Management of Adults With Congenital Heart Disease): developed in Collaboration with the American Society of Echocardiography, Heart Rhythm Society, International Society for Adult Congenital Heart Disease, Society for Cardiovascular Angiography and Interventions, and Society of Thoracic Surgeons. Circulation 2008;118:e714-833.

17 Baumgartner $\mathrm{H}$, Hung J, Bermejo J, et al. Echocardiographic assessment of valve stenosis: EAE/ASE recommendations for clinical practice. J Am Soc Echocardiogr 2009;22:1-23.

18 Lancellotti P, Tribouilloy C, Hagendorff A, et al. Recommendations for the echocardiographic assessment of native valvular regurgitation: an executive summary from the European Association of Cardiovascular Imaging. Eur Heart $J$ Cardiovasc Imaging 2013;14:611-44.

19 Buber J, Assenza GE, Huang A, et al. Durability of large diameter right ventricular outflow tract conduits in adults with congenital heart disease. Int I Cardiol 2014; 175:455-63.

20 Yemets IM, Williams WG, Webb GD, et al. Pulmonary valve replacement late after repair of tetralogy of Fallot. Ann Thorac Surg 1997;64:526-30.

21 Tweddell JS, Pelech AN, Frommelt PC, et al. Factors affecting longevity of homograft valves used in right ventricular outflow tract reconstruction for congenital heart disease. Circulation 2000;102:lii-130-5.

22 Wells WJ, Arroyo H Jr, Bremner RM, et al. Homograft conduit failure in infants is not due to somatic outgrowth. J Thorac Cardiovasc Surg 2002;124:88-96.

23 Schorn K, Yankah AC, Alexi-Meskhishvili V, et al. Risk factors for early degeneration of allografts in pulmonary circulation. Eur J Cardiothorac Surg 1997;11:62-9.

24 Koolbergen DR, Hazekamp MG, de Heer E, et al. The pathology of fresh and cryopreserved homograft heart valves: an analysis of forty explanted homograft valves. J Thorac Cardiovasc Surg 2002;124:689-97.

25 Dave $H$, Mueggler 0 , Comber $M$, et al. Risk factor analysis of 170 single-institutional contegra implantations in pulmonary position. Ann Thorac Surg 2011;91:195-203.

26 Urso S, Rega F, Meuris B, et al. The Contegra conduit in the right ventricular outflow tract is an independent risk factor for graft replacement. Eur I Cardiothorac Surg 2011;40:603-9.

27 Jang W, Kim YJ, Choi K, et al. Mid-term results of bioprosthetic pulmonary valve replacement in pulmonary regurgitation after tetralogy of Fallot repair. Eur $J$ Cardiothorac Surg 2012;42:e1-8.

28 Hunter RM, Isaac M, Frigiola A, et al. Lifetime costs and outcomes of repair of tetralogy of Fallot compared to natural progression of the disease: great ormond street hospital cohort. PLOS ONE 2013;8:e59734. 